\title{
Health Promotion opportunities for Auxiliary Nurse Midwives in Nepal
}

Preeti K Mahato ${ }^{1 *}$, Pramod R Regmi ${ }^{1-2}$, Elizabeth Waithaka ${ }^{1}$, Edwin R van Teijlingen ${ }^{1,3-4}$, Padam P Simkhada ${ }^{3-5}$, Catherine Angell ${ }^{1}$

${ }^{1}$ Faculty of Health \& Social Sciences, Bournemouth University, Bournemouth, UK

${ }^{2}$ Chitwan Medical College, Tribhuvan University, Nepal

${ }^{3}$ Manmohan Memorial Institute of Health Sciences, Tribhuvan University, Nepal

${ }^{4}$ Nobel College, Pokhara University, Nepal.

${ }^{5}$ Public Health Institute, Liverpool John Moores University, Liverpool, UK

\section{Received:}

28 August 2017

Revised:

21 November 2017

Accepted:

16 January 2018

${ }^{*}$ Corresponding author

pmahato@bournemouth.

ac.uk

Faculty of Health \&

Social Sciences, Bour-

nemouth University,

Bournemouth, UK

\begin{abstract}
Health promotion moves beyond changing the health-related knowledge, attitudes and behaviour of individuals and covers a wide range of social and environmental interventions. Nepal has also introduced health promotion policies targeting health education, information and communication. Auxiliary Nurse Midwives (ANMs), who are responsible for delivering primary care maternity services, especially in birthing centres located in rural areas of Nepal, also have an important role to play in the promotion of women's health during pregnancy, intra-partum and post-partum. However, in the present context, health promotion provided by ANMs in Nepal is confined mostly to health education and behavioural-change communication. There are a range of health promotion activities relevant to maternity care and midwifery that ANMs can practice even in low-technology rural birthing centres. Such health promotion offers an opportunity to move away from a very medical and behavioural model to a more empowering one in order to prevent health problems in a cost-effective way.
\end{abstract}

Keywords: midwifery, maternity care, South Asia, health education

Tweetable Abstract: Health promotion offers an opportunity for ANMs to move away from a medical and behavioral model to a more empowering one.

\section{Introduction}

Conventionally, health promotion is 'a process of enabling people to increase control over, and improve their health. It moves beyond a focus on individual health and behaviour towards a wide range of social and environmental interventions' [1]. Health promotion, therefore, is not only about disease prevention but also general health improvement, protection and education.

Auxiliary Nurse Midwives (ANMs) provide much of the primary care maternity services in Nepal, delivering care especially at birthing centres in rural areas. ANMs provide antenatal care (ANC), assistance at childbirth and postnatal care, as well as more general sexual and reproductive health care [2]. Midwives are promotors of health by their nature of profession. ANMs have 18 months of pre-service training in nursing and midwifery after ten years of schooling. The ANM curriculum is limited to conducting and managing normal deliveries and has less emphasis on promoting the health of pregnant and post-partum women [3] and there is little mention of health promotion activities that ANMs can carry out in their daily work. This article discusses about the role of ANMs in the field of maternal and child health in Nepal and the different health promotion opportunities available to them.

\section{Health promotion in Nepal}

Internationally, health promotion came into limelight with the Ottawa Charter in 1986. The first International Conference on Health Promotion in Canada notably led to the Ottawa Charter for Health Promotion. The Ottawa Charter identifies five important health promotion approaches: building healthy public policy, creating supportive environments, strengthening community actions, developing personal skills and reorienting health services [4]. These five areas of action strongly emphasize the social determinants of health and equity of distribution of health.

Being a signatory to the Ottawa Charter, Nepal introduced health promotion policies as early as 1991. The National Health Policy (1991) recognized promotive nature of primary health care by extending the system to the rural population. This was supported by establishing the National Health Education, Information and Communication Centre (NHEICC) in 1993, a governmental organization that supports health promotion [5]. The National Health Policy 2071 (2014) sets as one of its objectives the promotion of public health, emphasizing 
health education, information and communication, as well as protecting people's fundamental right to health information [6]. Interventions carried over the years by NHEICC resulted into an increased awareness evidenced by increased immunisation coverage and increased demand for health services. However, promotive actions and policies along with a preventive component are yet to be adequately integrated into the mainstream health services. The Government of Nepal (GoN) and various Non-Government Organizations (NGOs) including community-based organizations (CBOs) have been offering health promotion on a spectrum of issues, including family planning; maternal, neonatal and child health; adolescents' sexual and reproductive health.

\section{The role of Auxiliary Nurse Midwives in health promotion}

ANMs are trained to assist normal deliveries, identify complications and refer women to more specialist care. They are mostly deployed in rural Nepal where there is a lack of proper health care facilities [7] but some are deployed in hospital settings in urban areas. It is worth noting that Nepal does not currently recognize midwifery as a separate profession from nursing $[8,9]$.

A primary care model is thought to be best for most of the women of childbearing age and newborn infants who are healthy and at low risk of complications [10]. The primary care model of childbirth includes preventative measures, promotion of normal physiologic labour and birth, detection of complications, assessing medical care and that the carrying out of emergency measures are the scope of midwifery practice and are aligned with the competencies for basic midwifery practice [11]. The core element of midwifery care is providing care and support that promotes normal biopsychosocial processes, optimizes labour, birth, breastfeeding and attachment [10], thus, adhering to a more social model of childbirth [12].

Health promotion is considered as a key component of nursing and midwifery. ANMs in rural birthing centres clearly have a role in promoting the healthcare of women during pregnancy, as well as the intra-partum and post-partum periods. Health promotion is a core competency of a midwife and not an extended role [13]. The role of midwives in health promotion is being extended to deal with issues related to teenage pregnancy, smoking cessation, breastfeeding, domestic violence, mental health promotion, sexual health promotion and so on [13, 14]. Interestingly, the ANM curriculum in Nepal [2] pays attention to health education but there is little information on other health promotion approaches. Box 1 lists health education/promotion activities mentioned in the ANM curriculum [3]. Also, there is a lack of job description to ANMs to practice which further limits the health promotion activities performed by this cadre of health professionals [9]. It has been found that the term 'health promotion' is often been used interchangeably with health education but health promotion is so much more than just educating women and communities about their health [13]. Health education is considered as a part of health promotion and its effectiveness will be enhanced if there is a supportive environment established by a healthy public policy [15]. Although the National Safe Motherhood Program established in 1997 outlined many health promotion goals including the expansion of delivery by skilled birth attendant (SBA), basic and comprehensive obstetric care services at all levels, the role of ANMs in health promotion is not clearly defined [5]. Thus, it seems as though the ANMs don't know their role as health promoters.

\section{Box 1: Health promotion activities mentioned in ANM curriculum}

1. Midwifery " $\mathrm{A}$ "

Identify needs of pregnant women and give appropriate health information and education to mother and family in order to ensure healthy readiness of pregnancy, safe delivery and complication.

Needs of pregnant women:

- Nutrition

- Immunisation (Tetanus toxoid/deworming)

- Antenatal check-up

- Rest, sleep, and exercise

- Sex education

- Birth preparedness and complication readiness: Financial arrangement, transportation, food, clothing, person for blood donation

- Health education on above mentioned points plus breast feeding, personal hygiene, danger signs of pregnancy and counselling on postpartum family planning.

2. Midwifery "B"

Teach and counsel the mother, family and group to ensure safe delivery care to a new born, recognizing danger signs and action to be taken as needed.

Care for women in labour and during the birth according to the nursing process in any given situation.

Immediate administration of vitamin A to mother after delivery.

Care to women and newborn

- Care of immediate newborn baby

- Provide breast feeding immediately after birth 
- Prevent baby from hypothermia, accident
- Provide nursing care to mother
3. Midwifery "C"
Describe the care and management of puerperium including health education to mother and family
Infection prevention
- Nutrition and balance diet
- General cleanliness
- Perineal care
- Breast care
Promotion of uterus involution
- Immediate breast feeding
- Postnatal exercise
- Early ambulation
Psychological support
- Psychological preparation to adjust the motherhood
- Psychosis blue's
Discuss about exclusive breastfeeding
- Promote breastfeeding within an hour of birth
- Advantages of breastfeeding, positioning/attachment
Difficulties in breastfeeding
- Expression of breast milk and cup feeding
- Suppression of lactation

Health promotion activities carried by ANMs in the rural of Nepal is similar to those performed by midwives in Ghana and includes weight management; infection prevention; personal hygiene; family planning; and counselling on harmful substances such as alcohol, drugs and tobacco [16]. As the determinants of health are multifaceted, it is important that health promotion strategies should not only focus on changing individual behaviour but also on social and environmental factors [15]. In a traditional society such as Nepal, women have less control over decisions related to birth, for example, decision to attend ANC [17], therefore it is important that SBAs work in partnership with mothers and families especially mothers-in-law thus facilitating decisions about the care they need [14]. Promotion of inter-sectoral collaboration as stressed by National Safe Motherhood Program needs to be given more importance [5]. In addition, apart from being multidisciplinary, health promotion activities should also be evidence-based and theory-based [18]. In neighbouring India unlike Nepal, the role of ANMs in maternal health promotion is well established [19]. The promotion of wellbeing during pregnancy and universal immunisation in rural areas falls largely within the remit of ANMs [20]. The National Rural Health Mission (NRHM) acknowledges the significant health promoting role of ANMs at the interface of safe motherhood services in rural communities across India [21]. In the United Kingdom, the midwives have an important role in public health and as health promotors as they are key health personnel involved during pregnancy, childbirth and post-partum period of women. These midwives have a special place in their local communities and are ideally situated to help develop community support networks with women and their families [13].

Health promotion activities in Nepal mostly include health education, awareness generation and behaviour change communication rather than touching upon broader health promotion activities [22]. It is important that health promotional interventions be targeted for women, their husbands and family members especially mothers-in-law, which can be effectively done by ANMs in Nepal. Considering community-based needs assessments and incorporating diverse/changing needs of local communities through best use of existing resources is important while planning health promotion activities [18]. In order to make health promotion successful, it requires participation of all stakeholders including government, social services, education, employment and service users [23]. Co-ordination between government and other agencies for conducting health promotion activities is thus seen necessary along with focusing on social and empowerment approach rather than only stressing the medical, educational and behavioural approaches to health promotion [22]. In addition, a list of effective practices in health promotion appropriate for ANMs, over and above those mentioned in their curriculum, can be found in Box 2 This list was distilled from 461 systematic reviews to identify high quality, up-to-date evidence on the effectiveness of specific practices in maternal and newborn care relevant to the scope of midwifery [24]. There are few more activities suitable for ANMs working at birthing centres in rural parts as elaborated by Bowden and Manning [13]. For example, in sexual health promotion, they can provide private, confidential and comfortable environments to facilitate initiation of discussion and open and honest disclosure of sexual health concerns. Similarly, ANMs can be active in primary, secondary and tertiary mental health promotion by allowing mental issues to be normalized and more openly discussed with women and their families. In this way, women can be diagnosed earlier and referred on where necessary. In case of domestic violence ANMs play a crucial role in identifying pregnant women who are at risk of domestic 
violence. However, it must be recognized that there limited interventions possible to aid women in rural traditional communities.

Box 2: Effective health promotion relevant to midwifery care (adapted from Renfrew et. al 2014)

Effective practices in health promotion and promoting normal birth

- Insecticide treated nets for the prevention of malaria in pregnancy

- Specific advice to increase dietary energy and protein intakes or energy and protein supplementation in pregnancy

- Interventions to prevent smoking during pregnancy

- Peer support to promote breastfeeding initiation

- Supplementation with folic acid for women $<12$ weeks pregnant or pre-pregnant for prevention of neural tube defects

- Daily universal oral supplementation or intermittent supplementation with iron or iron and folic acid during pregnancy for the im

provement of maternal health

- Multiple micronutrient supplementations during pregnancy

- Skin-to-skin mother-baby contact within $24 \mathrm{~h}$ of birth

- Support for mothers to initiate breastfeeding within 1 (one) hour of birth

- Topical treatments for vaginal candidiasis in pregnancy

\section{Conclusion}

Health promotion activities by various health professional including ANMs are largely focused on health education and behavioural change strategies in Nepal. Involving of all sectors including service users and co-ordination between government and other agencies is necessary while planning for health promotion activities. There are a range of health promotion activities relevant to midwifery that ANMs can do even in technology limited rural birthing centres, apart from those mentioned in the ANM curriculum which are more or less educational. This health promotion offers an opportunity to move away from a very medical and behavioural model of health promotion to a more empowering one. Therefore, there is a chance to empower pregnant women and new mothers using health promotion in an effective way to help prevent future health problems in a cost-effective way.

\section{Competing interests}

The authors declare that they have no competing interests.

\section{Funding}

This work received no specific grant from any funding agency in the public, commercial, or not-for-profit sectors. The first author received a Civil Society Scholar Award (CSSA) from the Open Society Foundations.

\section{References}

1. World Health Organization. Health Promotion. Accessed 10 March 2017. http://www.who.int/topics/health_promotion/en/

2. United Nations Population Fund, International Confederation of Midwives, World Health Organization. State of the World's Midwifery report for 2014: 'A universal pathway. A woman's right to health' New York; UNFPA: 2014.

3. Vocational Training CDD. Auxiliary Nursing Midwifery (Post-SLC Program); 2014. Accessed 10 February 2016. http://ctevt.org.np/files/curriculum/TSLC/ANM\%20 Post\%20SLC\%20curriculum\%20Final\%203.24-2014.pdf.

4. World Health Organization. Ottawa Charter for health promotion: an international conference on health promotion, the move towards a new public health. 17-21

November. Geneva: World Health Organization; 1986. http://www.mohp.gov.np/index.php/publication-1/policy.

5. Ministry of Health and Population. Annual Report 2070/71 (2013/2014). Kathmandu: Department of Health Services, Ministry of Health and Population; 2013.

6. Ministry of Health and Population. National Health Policy 2014/15. Department of Health Services. Kathmandu; 2014. Accessed 10 February 2016.

7. Tamang, L. (2011). Birth and the establishment of a professional organization in Nepal. Midwifery Today Int Midwife 2011; 99: 53-4, 68-9.

8. Bogren, M., Bajracharya, K., Berg, M., Erlandsson, K., Ireland, J., Simkhada, P., et al. Nepal needs midwifery. J Manmohan Mem Inst Health Sci 2013; 1(2),41-4.

9. Bogren, M.U., van Teijlingen, E. \& Berg, M. Where midwives are not yet recognised: a feasibility study of professional midwives in Nepal. Midwifery 2013; 29 (10): 1103-9.

10. Sakala, C. \& Newburn, M. Meeting needs of childbearing women and newborn infants through strengthened midwifery. Lancet 2014; 384 (9948): e39-e40.

11. Fullerton, J.T., Thompson, J.B. \& Severino, R. The International Confederation of Midwives Essential Competencies for Basic Midwifery Practice. An update study: 2009-2010. Midwifery 2011; 27 (4): 399-408.

12. MacKenzie Bryers, H. \& van Teijlingen, E. Risk, theory, social and medical models: A critical analysis of the concept of risk in maternity care. Midwifery 2010; 26 (5): 488-96.

13. Bowden, J. \& Manning, V. Health Promotion in Midwifery 2nd edition, Principles and practice; Hodder Arnold Publication. London: 2006.

14. Beldon, A. \& Crozier, S. (2005). Health promotion in pregnancy: the role of the midwife. J R Soc Promot Health 2005; 125 (5): $216-20$.

15. Norton, L. Health promotion and health education: what role should the nurse adopt in practice? J Adv Nurs 1998; 28 (6): 1269-75. 
16. Owusu-Addo, E. Midwives' perceptions and experiences of health promotion practice in Ghana. Glob Health Promot 2015; 22 (3): 4-14.

17. Simkhada, B., Porter, M.A. \& van Teijlingen, E. The role of mothers-in-law in antenatal care decision-making in Nepal: a qualitative study. BMC Pregnancy Childbirth 2010; 10: 34, doi: 10.1186/1471-2393-10-34.

18. van Teijlingen, E., Simkhada, P., Stephens, J., Simkhada, B., Rogers, S.W. \& Sharma, S. (2012). Making the best use of all resources: developing a health intervention in rural Nepal. Health Renaissance 2012; 10 (3): 229-35.

19. Kumar, A. \& Mahapatro, M. The cutting edge in the blunt space: an anthropological construct of auxiliary nurse midwives' social world in the community. H Low Resour Settings 2013; 1: e10, doi: 10.4081/hls.2013.e10

20. Malik, G. Role of auxiliary nurse midwives in National Rural Health Mission. The Nurs J India 2009; 100 (4): 88-90.

21. Government of India. National Rural Health Mission Report. New Delhi: Government of India; 2005.

22. Sharma, A., Tuladhar, G., Dhungel, A., Padmadharini, van Teijlingen, E., \& Simkhada P. Health Promotion: A review of policies and practices in Nepal. Public Health Perspect 2015; 5(2).

23. World Health Organization, 1984. Health promotion: a discussion document on the concept and principles: summary report of the Working Group on Concept and Principles of Health Promotion, Copenhagen, 9-13 July 1984.

24. Renfrew, M.J., McFadden, A., Bastos, M.H, Campbell, J., Channon, A.A., Cheung, N.F., et al. Midwifery and quality care: findings from a new evidence-informed framework for maternal and newborn care. Lancet 2014; 384 (9948): 1129-45. 